\title{
Mycobacteria: Selection of Transplant Candidates and Post-lung Transplant Outcomes
}

\author{
Daniel Z.P. Friedman, MD ${ }^{1,2}$ Karen Doucette, MD $^{1}$ \\ ${ }^{1}$ Division of Infectious Diseases, Department of Medicine, University \\ of Alberta, Edmonton, Alberta, Canada \\ 2 Division of Infectious Disease, Department of Medicine, Mayo Clinic, \\ Rochester, Minnesota
}

\begin{abstract}
Address for correspondence Daniel Z.P. Friedman, MD, Mayo Clinic, 200 First Street SW, Rochester, MN 55905

(e-mail: friedman.daniel@mayo.edu).
\end{abstract}

Semin Respir Crit Care Med 2021;42:460-470.

\author{
Abstract \\ Keywords \\ - tuberculosis \\ - leprosy \\ - nontuberculous \\ mycobacteria \\ - Mycobacterium \\ abscessus \\ - Mycobacterium \\ chelonae \\ - Mycobacterium avium \\ complex
}

Mycobacterium is a large, clinically relevant bacterial genus made up of the agents of tuberculosis and leprosy and hundreds of species of saprophytic nontuberculous mycobacteria (NTM). Pathogenicity, clinical presentation, epidemiology, and antimicrobial susceptibilities are exceptionally diverse between species. Patients with endstage lung disease and recipients of lung transplants are at a higher risk of developing NTM colonization and disease and of severe manifestations and outcomes of tuberculosis. Data from the past three decades have increased our knowledge of these infections in lung transplant recipients. Still, there are knowledge gaps to be addressed to further our understanding of risk factors and optimal treatments for mycobacterial infections in this population.
Lung transplantation provides a therapeutic option for many patients with end-stage lung disease. The outcomes of lung transplantation continue to improve due to optimized pretransplant patient selection; novel surgical techniques, including ex vivo lung perfusion; and a better understanding of strategies to prevent rejection. ${ }^{1}$ Increasing numbers of successful lung transplants and improved longevity of recipients have presented new challenges with infections and chronic lung allograft dysfunction (CLAD).

Of significant importance is the impact of mycobacterial infections on lung transplant candidates and recipients. In 2019, more than 10 million people worldwide were diagnosed with active tuberculosis (TB), and many more are silently harboring latent infections. ${ }^{2}$ Migration significantly impacts both organ donors as well as transplant candidates and recipients with infections occurring even in low-prevalence countries. ${ }^{3}$ In regions with a low prevalence of TB, such as North America, Western Europe, and Australia, nontuberculous mycobacteria (NTM) are more frequently encountered and pose unique challenges in the lung transplant patient. ${ }^{4}$ Unlike TB, there is a lack of robust evidence for the optimal approach for managing NTM infections, and practices vary considerably between transplant centers. ${ }^{5}$

This review highlights recent studies and summarizes important findings of tuberculous and NTM infections' impact on lung transplant candidate selection and recipient outcomes, focusing on NTM infections.

\section{Microbiology and Pathogenicity of Mycobacterium spp.}

The bacterial genus Mycobacterium is composed of more than 200 species that, despite sharing some common features, are heterogeneous in their pathogenicity, epidemiology, and management. As with other genera within the family Mycobacteriaceae, Mycobacterium spp. have a cell wall with a high mycolic acid content that resists decolorization with acid alcohols and provides the characteristic positive acidfast staining. These bacteria are aerobic, non-spore-forming, and weak gram-positive bacilli. ${ }^{6}$
Issue Theme Lung Transplantation: Controversies and Evolving Concepts; Guest Editors: John A. Belperio, MD, and Scott M. Palmer, MD (c) 2021. Thieme. All rights reserved. Thieme Medical Publishers, Inc., 333 Seventh Avenue, 18th Floor, New York, NY 10001, USA 
The genus is further divided into several species complexes. The most well-recognized, the Mycobacterium tuberculosis (MTB) complex, comprises a group of pathogenic bacteria that cause TB in humans and other mammals. The most commonly implicated human pathogens include $M$. tuberculosis, M. bovis bacillus Calmette-Guérin (BCG) strain, $M$. africanum, and $M$. canetti. Zoophilic pathogens include $M$. bovis, M. pinnipedii, M. caprae, and M. microti. These are primarily transmitted by respiratory aerosols between persons or animals; although these are not considered sapronotic, species of the MTB complex have been recovered from environmental surveys. ${ }^{7,8}$ of global importance, another group of pathogenic mycobacterial species includes the three causative species of leprosy. M. leprae is the most commonly implicated organism, with sporadic leprosy cases being caused by M. lepromatosis. M. lepraemurium is the cause of leprosy in murine hosts. ${ }^{9}$

The other hundreds of mycobacterial species not included in these complexes are known collectively as NTM. NTM are ubiquitous environmental bacteria that, for the most part, are opportunistic pathogens or commensal bacteria, although exceptions do exist. To date, more than 150 NTM species have been recognized, and due to the advent of molecular techniques, such as 16S rRNA and heat shock protein sequencing, new species are being rapidly identified. $^{10,11}$

One of the first methods of characterizing NTM species dates back to 1959 and focuses on colony growth characteristics. This Runyon classification categorizes species by the colonies' growth rate, morphology, and pigmentation pattern. While newer molecular diagnostic techniques are increasingly utilized to speciate NTM, the speed of colony growth remains a significant clinical distinction. Rapidly growing mycobacteria (RGM), such as M. abscessus, M. fortuitum, and M. chelonae, typically produce visible colonies on solid media within 7 days, whereas slowly growing mycobacteria (SGM) may take up to 2 to 3 weeks. Common SGM species include those in the M. avium complex (MAC; $M$. avium, $M$. intracellulare, $M$. chimera, etc.), M. kansasii, $M$. xenopi, and M. haemophilum. Intermediate-growing mycobacteria, for example, M. marinum and $M$. gordonae, represent a small subgroup of SGM that requires anywhere from 7 to 10 days for colony production. Although the ability for a colony to produce pigment in both light and dark environments (scotochromogens), in light only (photochromogens), or not at all (nonchromogens), may help further differentiate mycobacterial species, this property is seldom used to identify species in modern microbiology laboratories.

The virulence factors and reasons for variable pathogenicity of NTM species are not entirely understood, although studies have identified which species are more likely to produce invasive pulmonary disease. In a multicenter Belgian cohort of 384 patients with pulmonary NTM infections, Vande Weygaerde and colleagues evaluated the clinical significance of individual species on the development of NTM disease. ${ }^{12}$ They found that more than $60 \%$ of those infected with $M$. abscessus, M. malmoense, $M$. intracellulare, and M. kansasii had disease. M. gordonae was among the most common nonpathogenic species and almost exclusively represented colonization. M. fortuitum was occasionally implicated in pulmonary disease, whereas M. avium and M. xenopi caused pulmonary disease approximately $50 \%$ of the time.

Some species, such as M. abscessus and M. avium, display two colonial morphotypes. The smooth morphotype has been associated with increased bacterial motility and biofilm formation, allowing them to persist in the environment. The rough morphotype is associated with severe or disseminated infections, likely due to mechanisms that bypass macrophage activity, such as the ability to form extracellular cording and induction of strong humoral immune response. ${ }^{13}$ The ability to transition between these two morphotypes may, in part, explain some species' increased pathogenicity. ${ }^{13-16} M$. abscessus can also be highly resistant to macrophages' bactericidal activity, allowing it to persist and multiply intracellularly, unlike less pathogenic species, whose ability to divide and survive in a host is readily halted by the macrophage. ${ }^{14}$

\section{Tuberculosis}

\section{Latent Tuberculosis-Diagnostic and Therapeutic Challenges}

Active TB following solid-organ transplantation (SOT) can be challenging to diagnose and treat due to altered inflammatory response, atypical radiographic findings, and drug-drug interactions. TB is associated with mortality as high as 10 to $20 \%$, although rates are decreasing possibly due to heightened clinical suspicion and optimization of treatment approaches to minimize and manage drug-drug interactions. ${ }^{17,18}$ The American Society of Transplantation recommends that all transplant candidates be screened and treated for latent TB infection (LTBI) before transplant. ${ }^{19}$ As outlined in these guidelines, an assessment should include symptom inquiry, a review of epidemiological risk factors, chest radiography, and either a tuberculin skin test (TST) or interferongamma release assay (IGRA). The sensitivity of the TST and IGRA is affected by systemic immunosuppression or chronic disease, and there is a risk of false-negative results after transplant. ${ }^{20}$ No studies have investigated whether treatment of LTBI before versus after transplant is associated with better patient and graft outcomes, but the guidelines recommend treatment begin before transplant when possible. ${ }^{19}$ Improved treatment adherence is anticipated when therapy is completed before transplant, because of lower pill burden, less drug interactions, and better tolerability. ${ }^{21,22}$ First-line therapy for LTBI is either 9 months of isoniazid, 4 months of rifampin, or 3 months of combination therapy with isoniazid and rifapentine. The use of rifampin and rifapentine is best avoided after transplant due to interactions with several classes of drugs, including immunosuppressants (-Table $\mathbf{1}$ ). Because treatment is several months in duration, the urgency of transplant may preclude a full course before transplant, necessitating a change in treatment after transplant. ${ }^{23}$ If active disease has been ruled out, LTBI is not a contraindication for transplant. Still, if the candidate has not received a complete course of either first-line or alternative therapy, 
Table 1 Important drug-drug interactions between select antimycobacterials and immunosuppressants used after lung transplant

\begin{tabular}{|l|l|l|}
\hline Antimycobacterial & IS & Interactions $48,77-82,115,116$ \\
\hline $\begin{array}{l}\text { Aminoglycosides } \\
\text { Amikacin } \\
\text { Tobramycin }\end{array}$ & CsA, Tac & Increased risk of nephrotoxicity \\
\hline $\begin{array}{l}\text { Fluoroquinolones } \\
\text { Ciprofloxacin } \\
\text { Levofloxacin } \\
\text { Moxifloxacin }\end{array}$ & CsA, Tac, Eve, Sir & $\begin{array}{l}\text { Increase IS levels (ciprofloxacin }>\text { levofloxacin }>\text { moxifloxacin), } \\
\text { QTc prolongation }\end{array}$ \\
\cline { 2 - 3 } Linezolid & MMF, MPA, AZA, Eve, Sir & Increased risk of tendinopathy \\
\hline $\begin{array}{l}\text { Macrolides } \\
\text { Azithromycin } \\
\text { Clarithromycin }\end{array}$ & CsA, Tac, Eve, Sir & Increase IS levels (clarithromycin > azithromycin) \\
\hline $\begin{array}{l}\text { Rifamycins } \\
\text { Rifampin } \\
\text { Rifabutin }\end{array}$ & CsA, Tac, corticosteroids, & Decrease IS levels (rifampin >> rifabutin) \\
\hline $\begin{array}{l}\text { Trimethoprim- } \\
\text { sulfamethoxazole }\end{array}$ & MMF, MPA, AZA, Eve, Sir & Increased risk of cytopenia (especially thrombocytopenia) \\
\cline { 2 - 3 } & CsA, Tac & Increased risk of nephrotoxicity \\
\hline
\end{tabular}

Abbreviations: AZA, azathioprine; CsA, cyclosporine; Eve, everolimus; IS, immunosuppressant; MMF, mycophenolate mofetil; MPA, mycophenolic acid; Sir, sirolimus; Tac, tacrolimus.

they should be treated following transplant with a regimen that does not include rifamycins. As isoniazid monotherapy is longer in duration and associated with increased risk of hepatotoxicity, therapy with rifampin completed before transplant may be preferred when possible to optimize the likelihood of treatment completion.

Lung transplantation is almost exclusively the result of deceased donation, with rare cases of living lobar donation. Therefore, the donor's past history and risk factors for TB are often unknown or misremembered. A consensus document has been published to address the appropriate workup and utilization of organs from at-risk donors. ${ }^{3}$ Donors with radiographic abnormalities, known exposures to TB or residents of endemic areas, should be assessed for LTBI. However, there is insufficient time to perform TST, and the use of IGRA has not been validated in deceased donors and has been shown to yield significant rates of indeterminate results. ${ }^{24}$ Because there have been reports of transmission of inadequately treated LTBI from lung donors, which can result in reactivation and dissemination in the recipient, ${ }^{25-28}$ the offer of lungs from high-risk donors must be carefully reviewed, including a consideration of chemoprophylaxis in the recipient.

\section{Active Tuberculosis-When to Transplant and Posttransplant Outcomes}

Active TB in a transplant candidate is considered an absolute contraindication for transplant, given the risk for dissemination and poor graft and patient outcomes. ${ }^{17,23,29}$ However, the timing at which a patient with active TB can safely undergo transplantation has not been clearly defined. A recent review of five SOT recipients with an unknown diagnosis of active TB at the time of transplant (two liver, one heart, one lung, one kidney) demonstrated overall favorable outcomes despite two patients experiencing acute rejection episodes 1 month after transplantation. ${ }^{30}$ The lung transplant patient died 6 months after transplant from acute rejection; however, the implication of active $\mathrm{TB}$ in the patient's demise is uncertain.

A high index of suspicion is needed for a prompt diagnosis of TB following SOT. Following transplant, patients may present with atypical, nonspecific presentations and are at higher risk for disseminated infection. ${ }^{23,31}$ Even with pulmonary disease, fever and constitutional symptoms can be more apparent than dyspnea or cough, ${ }^{32}$ and in the case of lung transplant, pulmonary consolidations and cavitary lesions are not frequently seen on chest radiography. ${ }^{31}$

Recent reviews of TB and SOT recipients by Abad and Razonable have shed some light on this subject. ${ }^{26,31}$ of more than 2,000 patients, only $1.2 \%$ were lung transplant recipients, representing a prevalence of 0.96 cases per 100 lung transplant recipients. Almost half of the infections in lung recipients were donor derived, which was the highest proportion of all organ groups. The mortality rate was highest in lung transplant recipients at $25 \%$, compared with $23.8,20.3$, and $18.8 \%$ in heart, liver, and kidney recipients, respectively. In a retrospective review of 398 lung transplant recipients, use of azathioprine and everolimus was independently associated with developing TB after lung transplant. ${ }^{33}$ We believe that the association of TB with everolimus was likely confounded by the fact that everolimus was more commonly used in patients with comorbidities, such as renal failure, allograft rejection, and malignancies.

The standard first-line regimen for the treatment of susceptible active TB is an intensive phase with isoniazid, rifampin, pyrazinamide, and ethambutol for 2 months, followed by at least an additional 4 months of rifampin and 
isoniazid. ${ }^{19,34,35}$ The prolonged use of some of these agents following lung transplant is challenging due to drug interactions and toxicities ( $\mathbf{- T a b l e ~} \mathbf{1}$ ). Successful treatment with rifampin-based therapy after lung transplant has been reported, but requires very careful monitoring of calcineurin inhibitor levels and toxicity. ${ }^{36,37}$ To address the concern of rifampin use after transplant, rifabutin has been substituted to treat active TB following SOT with success. ${ }^{38,39}$

\section{Nontuberculous mycobacteria}

\section{Epidemiology}

The prevalence of NTM infections is likely underestimated, mainly due to asymptomatic colonization and nonuniversal requirements to report infections to public health authorities. ${ }^{40}$ There is significant variation in the geographic distribution of species globally, and this has been previously detailed in a review by Zweijpfenning and colleagues. ${ }^{41}$ Species in the M. avium complex are the most common species from clinical isolates worldwide and account for more than half of all infections in North America ${ }^{40-42}$; geographic variation also exists within this complex, with M. avium being more prevalent in Europe and the Americas, and $M$. intracellulare more dominant in Australia and South Africa. $^{42}$

North American data are primarily limited to publications from the United States (-Table 2). In a recent review of almost 6 million patients across the United States from 2009 to 2013 , Spaulding et al found $0.13 \%$ of patients had a positive respiratory culture for an NTM species. ${ }^{43}$ Canadian data report the annual rate of NTM isolation in the general population as 14.1 to 22.2 per $100,000 .{ }^{44-47}$ Rates of reported cases of NTM infections are increasing worldwide, likely due to growing awareness of NTM, improvement in current diagnostic techniques, increased use of immunosuppressive agents, and jurisdictions implementing decrease temperature of household water heaters. ${ }^{48-51}$

Most NTM species are nonpathogenic in patients with intact cell-mediated immunity. In immunocompetent hosts, pulmonary infection is the most common manifestation, with a propensity for those with structural airway disease. ${ }^{11}$ Infections of the skin and soft tissues in otherwise healthy adults typically require a mechanism of inoculation, such as a puncture wound ${ }^{52,53}$ or following surgery, particularly cosmetic surgery or surgeries related to medical tourism. ${ }^{54,55}$

Patients with cystic fibrosis (CF) and bronchiectasis comprise a population with more NTM infections, in part due to some centers routinely screening sputum of these patients for NTM and guidelines recommending all patients be assessed for NTM infection before transplant listing. ${ }^{29,56}$ In a multicenter cross-sectional study, $13 \%$ of patients with CF in the United States had NTM isolated from sputum. ${ }^{57}$ Subsequent studies have shown the prevalence as high as $28 \%$ in patients with $\mathrm{CF}$ and $10 \%$ in those with non-CF bronchiectasis. ${ }^{58}$ Research in zebra fish models suggest that the CF transmembrane conductance regulator (CFTR) protein plays a role in the neutrophilic response against $M$. abscessus and mutations of this gene may explain why patients with $\mathrm{CF}$ are at higher risk of infection compared with those with non-CF bronchiectasis. ${ }^{59,60}$ Individual factors associated with increased risk of pulmonary NTM infections in CF patients, particularly with $M$. abscessus, include concomitant allergic bronchopulmonary aspergillosis, airway colonization with Pseudomonas aeruginosa or Burkholderia cepacia, and chronic macrolide therapy. ${ }^{13,61}$ In one study, higher forced expiratory volume in 1 second (FEV1) was also associated with a higher probability of NTM recovery. ${ }^{57}$

A dysfunctional cell-mediated immune response is a nearly universal requirement for the development of disseminated infections, which became apparent during the acquired immunodeficiency syndrome (AIDS) pandemic of the 1980s-1990s when disseminated MAC infections were seen in patients with profound CD4-positive T-cell lymphopenia. ${ }^{62}$ Other recognized predispositions include prolonged use of tumor necrosis factor- $\alpha$ (TNF- $\alpha$ ) inhibitors, immunosuppression following solid organ and hematopoietic stem cell transplants, and genetic disruptions of the IL-12/interferon-gamma (IFN- $\gamma$ ) pathways. ${ }^{40,63}$ Although most IL-12/ IFN- $\gamma$ pathway defects are diagnosed in childhood, two diseases are becoming more recognized for their role in predisposing adults to extrapulmonary and disseminated NTM infections-GATA2 mutations and anti-IFN- $\gamma$ autoantibody syndrome, the latter being more common in adults of Southeast Asian descent. ${ }^{64,65}$ The recent global outbreak of $M$. chimaera infections in patients exposed to certain heatercooler units during open cardiovascular surgery has demonstrated that disseminated infections can occur in otherwise immunocompetent hosts. ${ }^{66}$

The prevalence of NTM infections in SOT recipients is higher than that in the general population, with the highest rates in heart and lung transplant recipients $(0.24-2.8 \%$ and 0.46-9\%, respectively). ${ }^{67-69}$ Rates among renal and liver transplant recipients are lower but have been reported as high as 0.16 to $0.38 \%$ and $0.04 \%$, respectively. ${ }^{67,68}$ Some identified variables associated with the development of infection following SOT include episodes of acute rejection, chronic kidney disease, and $\mathrm{CF}^{70}$

\section{Clinical Manifestations and Treatment}

Clinical manifestations of NTM infections are highly variable due to species-specific virulence factors, route of infection, and host immune status. ${ }^{71}$ Pleuropulmonary infections are the most common presentations in the general population and lung transplant recipients. ${ }^{67}$ Two classical radiographic patterns exist-apical fibrocavitary disease (-Fig. 1), which is common to patients with underlying structural lung disease (such as pneumoconiosis or chronic obstructive pulmonary disease), and nodular bronchiectatic disease. ${ }^{71}$ The latter has been seen with MAC infections typically in thin, middle-aged, Caucasian women without any clear predisposing factor. ${ }^{72,73}$ Lung transplant recipients are at particular risk for NTM lung infections due to a combination of factors, including immune suppression; local defects in the transplanted allograft resulting in 
Table 2 Geographic variation of common NTM species in North America

\begin{tabular}{|c|c|c|c|c|c|c|}
\hline \multirow[t]{2}{*}{ Region } & \multicolumn{5}{|c|}{ Species distribution, \% } & \multirow[t]{2}{*}{ Reference } \\
\hline & M. avium complex & $\begin{array}{l}\text { M. chelonae/ } \\
\text { abscessus }\end{array}$ & M. fortuitum & M. kansasii & M. xenopi & \\
\hline \multicolumn{7}{|l|}{ Canada } \\
\hline Ontario & $48.1-63.0$ & $2.1-5.7^{b}$ & $2.6-4.7^{b}$ & $1.3-1.9$ & $19.2-26.5$ & $45-47,97$ \\
\hline $\begin{array}{l}\text { Western Canada } \\
\text { (Alberta, British Columbia) }\end{array}$ & $54.9-69.8$ & $13.2-21.6$ & $5.2-7.8$ & $1.3-2.0$ & $0-1.3$ & 89,117 \\
\hline \multicolumn{7}{|l|}{ The United States } \\
\hline New England ${ }^{\mathrm{b}}$ & 86 & 4 & NR & NR & NR & 43 \\
\hline Middle Atlantic ${ }^{a}$ & $80.1-83.6$ & $5.0-12.1$ & $3.2-5.6$ & $2.4-5.5$ & $1.7-2.6$ & $43,118,119$ \\
\hline East South Central $^{\mathrm{a}}$ & 91 & 2 & NR & NR & NR & 43 \\
\hline South Atlantic ${ }^{\mathrm{a}}$ & $48.3-78.0$ & $9.0-17.9$ & 6.3 & 1.9 & NR & 43,120 \\
\hline West South Central ${ }^{\mathrm{a}}$ & 61 & 18 & NR & NR & NR & 43 \\
\hline Mountain $^{\mathrm{a}}$ & 80 & 4 & NR & NR & NR & 43 \\
\hline Pacific $^{\mathrm{a}}$ & $69.3-87.5$ & $3.0-20.0$ & $0.5-24$ & $0.5-5.5$ & $0-1.7$ & $43,119,121-125$ \\
\hline East North Central $^{\mathrm{a}}$ & 78 & 7 & NR & NR & NR & 43 \\
\hline West North Central ${ }^{a}$ & 64 & 10 & NR & NR & NR & 43 \\
\hline
\end{tabular}

Abbreviations: M., Mycobacterium; NR, not reported; NTM, nontuberculous mycobacteria.

an Marras et al, ${ }^{45} M$. chelonae, M. abscessus, and $M$. fortuitum were combined to $13.0 \%$.

${ }^{\text {b}}$ States included in each region are defined in Spaulding et al. ${ }^{43}$

abnormal ciliary function; bronchial devascularization, denervation, and lymphatic insufficiency posttransplant; propensity for pretransplant airway colonization with NTM; and the nature of the lung itself with constant environmental exposure.

Skin and soft-tissue infections are often associated with the RGM species, particularly following trauma or surgery. M. abscessus and M. chelonae may cause surgical-site infections that can be progressive and associated with high mortality. ${ }^{74}$ Except in lung transplant recipients, where they account for only $10 \%$ of NTM infections, cutaneous disease and surgical site infections are the predominant presentations following SOT and hematopoietic stem cell transplant (-Fig. 2)..$^{67,75,76}$

The signs and symptoms of disseminated infection are protean and nonspecific. These may include fever, night sweats, weight loss, lymphadenopathy, and diarrhea. ${ }^{48}$

A crucial step in managing NTM infections is determining whether the presence of infection represents colonization or disease that may require therapy. Isolation of species known to be pathogenic from sterile spaces or histopathologic evidence of invasive disease supports the decision to treat. Pulmonary infections can be challenging to categorize as colonization or disease, and therefore criteria have been established and published in guidelines to aid in decisionmaking. ${ }^{48,77,78}$ The diagnosis of pulmonary disease is supported by repeated, good-quality respiratory cultures, compatible symptoms or objective pulmonary dysfunction, and abnormal imaging ( - Table 3 ).

The choice and duration of therapy are dependent on the organism, in vitro susceptibility pattern (where validated), site of infection, and degree of immunosuppression. Although the details of treatment regimens are case-specific, some considerations are highlighted. ${ }^{48,77,78}$ The cornerstone of therapy includes the use of combination antimycobacterial therapy, often including macrolides, rifamycins, ethambutol, isoniazid, fluoroquinolones, linezolid, tetracyclines, or aminoglycosides, depending on the organism. The duration of antimicrobial treatment is generally months to years, depending on the site of infection and severity. Several of these antimicrobials, particularly rifamycins, can interact with immunosuppressive therapy, and therefore close attention to immunosuppressant levels is required. Aminoglycosides can also potentiate calcineurin-associated nephrotoxicity ${ }^{79}$ and linezolid has been associated with higher rates of cytopenias, especially thrombocytopenia, in SOT recipients. ${ }^{80,81}$ Tendinopathies occur at a higher rate with fluoroquinolones in SOT recipients, especially those on chronic corticosteroids $(- \text { Table } \mathbf{1})^{82}$

Surgical debridement or resection should also be considered as an adjunct especially in cases of localized skin and soft-tissue infections. ${ }^{78}$ In SOT recipients and others with iatrogenic immunosuppression, decreasing the degree of immunosuppression may be required to effectively eradicate complicated infections, with particular consideration for monitoring signs of allograft dysfunction or rejection. ${ }^{83}$

The use of bacteriophages is being investigated as a novel therapeutic option for the management of multidrug-resistant (MDR) organisms. Bacteriophages are viruses that are selected from banks with natural occurring activity to attack 

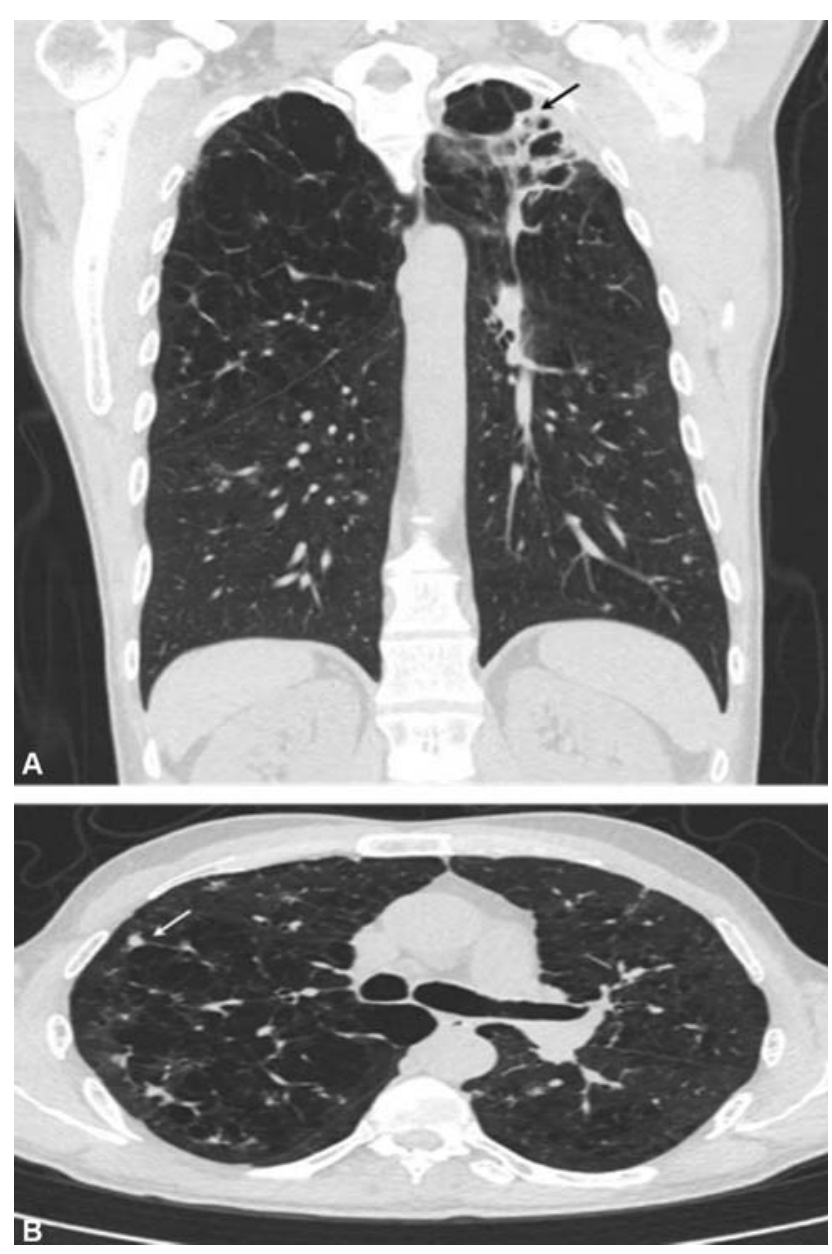

Fig. 1 High-resolution computed tomographic scan of a man with severe emphysema, bronchiectasis, and fibrocavitary M. xenopi pulmonary disease. There is a large left upper lobe fibrocavitary lesion (black arrow) seen on the coronal film (A). Spiculated subcentimeter nodules (white arrow) are seen in the right upper lobe seen on axial film (B).

a specific bacterial strain. In vitro studies and clinical reports show promise in SOT recipients, patients with ventricular assist devices, and nontransplant candidates. ${ }^{84}$ To date, there have been four reported cases of their use in treating infections with MDR organisms in lung transplant recipients. ${ }^{85,86}$ One of these patients was a 15 -year-old girl with $\mathrm{CF}$ and disseminated M. abscessus infection. After receiving over 8 years of antimycobacterials, she received a bilateral lung transplant. Following transplant, her antimicrobials were stopped due to side effects and she developed worsening pulmonary and surgical site infections. She was treated with a prolonged course of intravenous and topical bacteriophage-containing solutions in combination with resuming her antimicrobials and she experienced clinical and bacteriologic improvement over the subsequent 6 months. ${ }^{86,87}$ Although further research for its use in mycobacterial infections before and after transplant is needed, bacteriophage therapy seems to be a potential option for challenging NTM infections and reducing cumulative antimicrobial exposure.
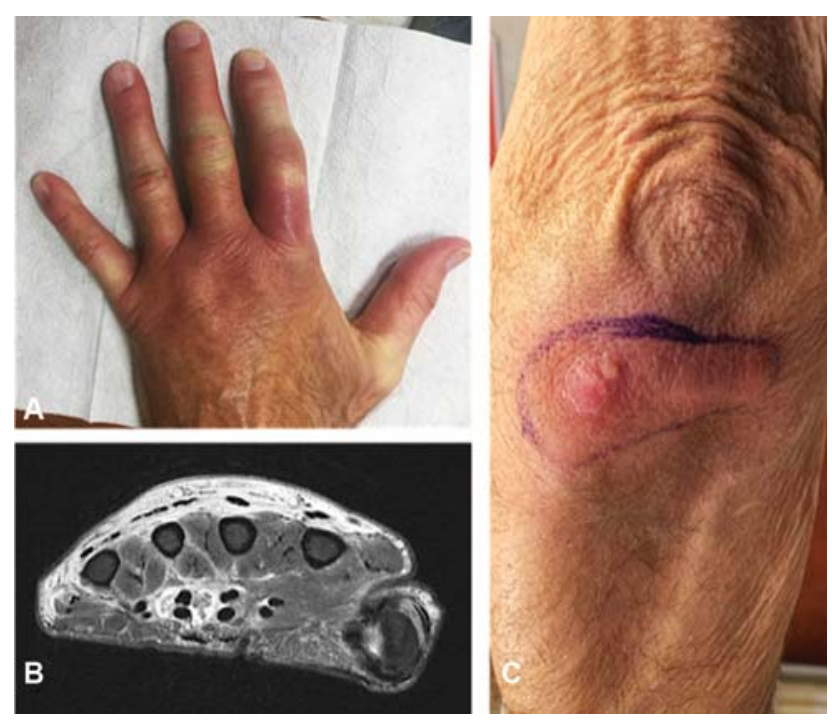

Fig. 2 M. chelonae musculocutaneous infection in a solid-organ transplant. Left hand swelling and cellulitis $(\mathrm{A})$ are noted with corresponding flexor tenosynovitis and associated lumbrical myositis of the third to fifth digits seen on gadolinium-enhanced magnetic resonant scan (B). The infection progressed to the ipsilateral elbow with nodule and abscess formation (C).

\section{Pretransplant Evaluation and Posttransplant Outcomes}

Although active infection with $M$. tuberculosis is considered an absolute contraindication for transplant, pursuing transplant in patients with NTM colonization and disease is a more nuanced decision. ${ }^{88}$ Data on candidate selection and predicting outcomes are sparse, conflicting, and primarily limited to case reports or series.

Reports are varying regarding the impact of NTM infections on patient survival and graft outcomes following lung transplantation. Several cohort studies have shown that NTM infections, including both colonization and disease, are associated with as high as a twofold increase in mortality following transplant; however, no significant association was found between infection and development of CLAD in these studies. ${ }^{89-92}$ Longworth et al specifically compared SOT recipients with NTM disease diagnosed in the first year with matched controls and showed a significant increase in 3-year mortality. ${ }^{91}$ This increased risk was similar between all NTM species.

On the other hand, several studies have found no increase in mortality associated with NTM infections following transplant. In a 15-year analysis of 237 lung transplant recipients, Knoll et al found no association with NTM infection and increased posttransplant mortality. ${ }^{93}$ George et al found in their cohort of 553 lung transplant recipients that those with NTM infection did not experience increased mortality, except in a small subset of patients with active disease. ${ }^{94}$ In another cohort of 208 lung transplant recipients, those with bronchopulmonary NTM disease posttransplant had a higher hazard of bronchiolitis obliterans syndrome (BOS), but not mortality. ${ }^{95}$

Following lung transplant, an algorithm based on the explanted lung pathology and microbiology has been 
Table 3 ATS/IDSA criteria for pulmonary NTM disease $e^{10,48}$

\begin{tabular}{|l|}
\hline Clinical (all criteria required) \\
\hline 1. Pulmonary (chronic cough, dyspnea, or hemoptysis) or systemic symptoms \\
2. Nodular or cavitary lesions on chest X-ray or multifocal nodular bronchiectasis on high-resolution CT scan \\
3. Exclusion of other diagnoses \\
\hline Microbiological (at least one criterion required) \\
1. Positive culture results of the same species from 2 separate expectorated sputums or \\
2. A positive culture from at least one bronchoscopic specimen or \\
3. Lung biopsy with histopathologic features consistent with mycobacterial infection with a positive culture for an NTM \\
species from the biopsy, sputum, or bronchoscopic specimen
\end{tabular}

Abbreviations: ATS, American Thoracic Society; IDSA, Infectious Disease Society of America; NTM, nontuberculous mycobacteria.

proposed by Hirama et al to help determine the antimicrobial approach of recipients with non-M. abscessus pulmonary NTM infection. ${ }^{96}$ Kabbani et al compared 148 lung and heart-lung transplant recipients with granulomata in the explanted lungs to matched controls without granulomatous pathology, finding that the presence of granulomata on explant (both necrotizing and nonnecrotizing) and positive mycobacterial cultures to be positive predictors for the development of posttransplant mycobacterial colonization and disease. However, neither had significant associations with patient survival. Interestingly, in this study, pretransplant NTM disease (including M. abscessus) and use of preventative therapy were not statistically significant risk factors. ${ }^{97}$ These findings support an algorithm for identifying patients early posttransplant who could benefit from more aggressive antimycobacterial therapy and highlight that pretransplant infection should not be an absolute contraindication to transplant.

\section{Mycobacterium abscessus and Other Rapidly Growing Mycobacteria}

Mycobacterium abscessus is one of the most challenging pathogens due to its high degree of antimicrobial resistance, its pathogenicity including propensity for biofilm formation, its increased prevalence among patients with $\mathrm{CF}$, and difficulty eradicating infection even with optimal therapy. ${ }^{13,14,98,99}$ For these reasons, the presence of $M$. abscessus is considered a contraindication to transplant in some centers. ${ }^{5,99,100}$ Life-threatening infections have occurred posttransplant in those with pretransplant colonization without invasive disease, and others with well-treated pulmonary disease have done well. Some centers require that patients infected with $M$. abscessus attain sputum smear negativity and be stable on antimycobacterial therapy before being listed for or undergoing transplantation. ${ }^{5,98,101}$

Posttransplant outcomes in those with M. abscessus infection are mixed. In a cohort of 1,301 lung transplant recipients, Hamad et al analyzed 22 cases of $M$. abscessus infection in the first year posttransplant, concluding that pulmonary disease was associated with a significant increase in 1-year mortality, but not BOS, compared with those who were colonized. ${ }^{90}$ In a small group of CF patients with $M$. abscessus lung disease following lung transplant, Perez et al found no difference in survival or graft outcomes compared with uninfected CF patients. ${ }^{102}$ Because of its propensity for severe and late-onset infections, some recommend therapy for over a year followed by chronic suppressive therapy to reduce the risk of early and late recurrent $M$. abscessus infections. $^{98}$

Until 1992, M. abscessus was considered within the same species complex as $M$. chelonae, and to date, some laboratories still have difficulties differentiating the two species. ${ }^{103}$ Before this distinction was made, several case reports of lateonset $M$. chelonae infections had been reported following thoracic transplant with severe or fatal outcomes. ${ }^{104,105}$ More recently, $M$. chelonae has been implicated in more localized cutaneous and musculoskeletal infections, which can be limb-threatening and recalcitrant. ${ }^{106}$

Posttransplant outcomes in patients with species other than MAC and M. abscessus are primarily limited to those published in case reports. ${ }^{76} \mathrm{M}$. kansasii airway colonization has been associated with favorable short-term outcomes following transplant. ${ }^{107}$ Four cases of cutaneous infection with $M$. haemophilum have been published, all having been treated to resolution with 17 to 42 months of combination rifampin- and macrolide-based therapy. ${ }^{108}$ Cases of surgical wound and localized cutaneous infections with non-M. abscessus species have been reported with successful outcomes following 6 to 9 months of multidrug therapy. $^{106,109}$

\section{Leprosy}

There have been no cases of leprosy in lung transplant recipients to date. Since the 1960 s, leprosy has rarely been reported, mainly in kidney transplant recipients, with few cases in liver and heart transplant recipients. The higher reported incidence in these organ groups is likely reflective of the higher rates of kidney, liver, and heart transplantation occurring globally, while lung transplantation tends to be more geographically restricted. Date et al reported nine cases of patients with leprosy diagnosed at various time points before and after kidney transplantation. ${ }^{110}$ of the patients who were followed up, all did well with resolution of cutaneous and neurologic symptoms, but several had late mortality from renal or liver dysfunction; mortality was not directly attributable to leprosy but likely was affected by long-term antileprous therapy. In SOT, tuberculoid leprosy is less common than lepromatous leprosy, likely due to immunosuppressant drugs that impair the Th1-cell-mediated 
immunity required for a tuberculoid inflammatory response. $^{110,111}$

There are only two reported cases of leprosy following thoracic organ transplantation, both in heart transplant recipients and both diagnosed as borderline lepromatous leprosy. One patient was a man from Louisiana with possible exposure to nine-banded armadillos (a known natural reservoir for M. leprae) by way of his dog. ${ }^{112}$ The second was an American man originally from India who presented 5 years after transplant with concomitant diagnoses of leprosy, firstepisode CMV viremia, and gastrointestinal cryptosporidiosis. ${ }^{113}$ Both patients had resolution of lesions with multidrug therapy, including dapsone and minocycline with either moxifloxacin or ethionamide. Although standard therapy for lepromatous leprosy includes clofazimine and rifampin, ${ }^{114}$ these were not used to avoid cardiac conduction abnormalities associated with clofazimine and drug interactions between rifampin and immunosuppressants.

Lessons learned from these non-lung transplant patients highlight strategies for management of leprosy that can be used if cases of either pauci- or multibacillary arise in a lung transplant recipient.

\section{Conclusion}

As the practice of lung transplantation continues to improve, it provides a valuable option to those suffering from chronic end-stage pulmonary diseases. The most common long-term risk of organ transplantation is that of infections associated with systemic immunosuppression. Although this risk can be mitigated, exposure to environmental and saprophytic organisms is often unavoidable. The advancements in the management of mycobacterial infections in lung transplant candidates and recipients have been the increasing recognition over the past decades, with the development of more thorough definitions and guidelines to direct therapy. We have a better yet incomplete understanding of the impact of NTM infections in lung transplant recipients. There is an ongoing need for more rigorous studies to better define durations of therapy, optimal surgical and perioperative approaches in those with NTM infection, and to enhance evidence-based transplant candidacy assessments for patients infected with NTM. As the number of transplants performed continues to climb worldwide, there is a need for multicenter collaboration to systematically study the effects of mycobacterial infections on transplant recipients and optimize management recommendations.

Conflict of Interest

None declared.

\section{References}

1 Meyer KC. Recent advances in lung transplantation. F1000 Res 2018;7:1684

2 World Health Organization. Global Tuberculosis Report 2020. 2020. Accessed January 4, 2021 at: https://apps.who.int/iris/bitstream/handle/10665/336069/9789240013131-eng.pdf
3 Morris MI, Daly JS, Blumberg E, et al. Diagnosis and management of tuberculosis in transplant donors: a donor-derived infections consensus conference report. Am J Transplant 2012;12(09): 2288-2300

4 Brode SK, Daley CL, Marras TK. The epidemiologic relationship between tuberculosis and non-tuberculous mycobacterial disease: a systematic review. Int J Tuberc Lung Dis 2014;18(11): 1370-1377

5 Tissot A, Thomas MF, Corris PA, Brodlie M. Nontuberculous mycobacteria infection and lung transplantation in cystic fibrosis: a worldwide survey of clinical practice. BMC Pulm Med 2018; 18(01):86

6 Forbes BA, Hall GS, Miller MB, et al. Practical guidance for clinical microbiology laboratories: mycobacteria. Clin Microbiol Rev 2018;31(02):e00038-e17

7 Martinez L, Verma R, Croda J, et al. Detection, survival and infectious potential of Mycobacterium tuberculosis in the environment: a review of the evidence and epidemiological implications. Eur Respir J 2019;53(06):1802302

8 Velayati AA, Farnia P, Mozafari M, et al. Identification and genotyping of Mycobacterium tuberculosis isolated from water and soil samples of a metropolitan city. Chest 2015;147(04): 1094-1102

9 Rojas-Espinosa O, Løvik M. Mycobacterium leprae and Mycobacterium lepraemurium infections in domestic and wild animals. Rev Sci Tech 2001;20(01):219-251

10 Daley CL, Iaccarino JM, Lange C, et al. Treatment of nontuberculous mycobacterial pulmonary disease: an official ATS/ERS/ESCMID/IDSA clinical practice guideline: executive summary. Clin Infect Dis 2020;71(04):e1-e36

11 Koh W-J. Nontuberculous mycobacteria-overview. Microbiol Spectr 2017;5(01):1-7

12 Vande Weygaerde Y, Cardinaels N, Bomans P, et al. Clinical relevance of pulmonary non-tuberculous mycobacterial isolates in three reference centres in Belgium: a multicentre retrospective analysis. BMC Infect Dis 2019;19(01):1061

13 Degiacomi G, Sammartino JC, Chiarelli LR, Riabova O, Makarov V, Pasca MR. Mycobacterium abscessus, an emerging and worrisome pathogen among cystic fibrosis patients. Int J Mol Sci 2019; 20(23):E5868

14 Roux A-L, Viljoen A, Bah A, et al. The distinct fate of smooth and rough Mycobacterium abscessus variants inside macrophages. Open Biol 2016;6(11):160185

15 Jönsson BE, Gilljam M, Lindblad A, Ridell M, Wold AE, WelinderOlsson C. Molecular epidemiology of Mycobacterium abscessus, with focus on cystic fibrosis. J Clin Microbiol 2007;45(05): 1497-1504

16 Faria S, Joao I, Jordao L. General overview on nontuberculous mycobacteria, biofilms, and human infection. J Pathogens 2015; 2015:809014

17 Torre-Cisneros J, Doblas A, Aguado JM, et al; Spanish Network for Research in Infectious Diseases. Tuberculosis after solid-organ transplant: incidence, risk factors, and clinical characteristics in the RESITRA (Spanish Network of Infection in Transplantation) cohort. Clin Infect Dis 2009;48(12):1657-1665

18 Mamishi S, Pourakbari B, Moradzadeh M, van Leeuwen WB, Mahmoudi S. Prevalence of active tuberculosis infection in transplant recipients: a systematic review and meta-analysis. Microb Pathog 2020;139:103894

19 Subramanian AK, Theodoropoulos NMInfectious Diseases Community of Practice of the American Society of Transplantation. Mycobacterium tuberculosis infections in solid organ transplantation: guidelines from the infectious diseases community of practice of the American Society of Transplantation. Clin Transplant 2019;33(09):e13513

20 Hadaya K, Bridevaux P-O, Roux-Lombard P, et al. Contribution of interferon- $\gamma$ release assays (IGRAs) to the diagnosis of latent 
tuberculosis infection after renal transplantation. Transplantation 2013;95(12):1485-1490

21 Horne DJ, Narita M, Spitters CL, Parimi S, Dodson S, Limaye AP. Challenging issues in tuberculosis in solid organ transplantation. Clin Infect Dis 2013;57(10):1473-1482

22 Sidhu A, Verma G, Humar A, Kumar D. Outcome of latent tuberculosis infection in solid organ transplant recipients over a 10-year period. Transplantation 2014;98(06):671-675

23 Subramanian AK. Tuberculosis in solid organ transplant candidates and recipients: current and future challenges. Curr Opin Infect Dis 2014;27(04):316-321

24 Tabarsi P, Yousefzadeh A, Najafizadeh K, et al. Performance of QuantiFERON TB Gold test in detecting latent tuberculosis infection in brain-dead organ donors in Iran: a brief report. Saudi J Kidney Dis Transpl 2014;25(06):1240-1243

25 Jensen TO, Darley DR, Goeman EE, Shaw K, Marriott DJ, Glanville AR. Donor-derived tuberculosis (TB): isoniazid-resistant TB transmitted from a lung transplant donor with inadequately treated latent infection. Transpl Infect Dis 2016;18(05):782-784

26 Abad CLR, Razonable RR. Donor derived Mycobacterium tuberculosis infection after solid-organ transplantation: a comprehensive review. Transpl Infect Dis 2018;20(05):e12971

27 Kumar D, Budev M, Koval C, Hellinger WC, Gordon SM, Tomford JW. Donor-derived tuberculosis (TB) infection in lung transplant despite following recommended algorithm. Am J Transplant 2013;13(08):2225-2226

28 Ridgeway AL, Warner GS, Phillips P, et al. Transmission of Mycobacterium tuberculosis to recipients of single lung transplants from the same donor. Am J Respir Crit Care Med 1996;153 (03):1166-1168

29 Weill D, Benden C, Corris PA, et al. A consensus document for the selection of lung transplant candidates: 2014-an update from the Pulmonary Transplantation Council of the International Society for Heart and Lung Transplantation. J Heart Lung Transplant 2015;34(01):1-15

30 Torre-Cisneros J, Castón JJ, Moreno J, et al. Tuberculosis in the transplant candidate: importance of early diagnosis and treatment. Transplantation 2004;77(09):1376-1380

31 Abad CLR, Razonable RR. Mycobacterium tuberculosis after solid organ transplantation: a review of more than 2000 cases. Clin Transplant 2018;32(06):e13259

32 Bodro M, Sabé N, Santín M, et al. Clinical features and outcomes of tuberculosis in solid organ transplant recipients. Transplant Proc 2012;44(09):2686-2689

33 Guirao-Arrabal E, Santos F, Redel-Montero J, et al. Risk of tuberculosis after lung transplantation: the value of pretransplant chest computed tomography and the impact of mTOR inhibitors and azathioprine use. Transpl Infect Dis 2016;18 (04):512-519

34 Menzies D, Elwood K. Canadian Tuberculosis Standards: Treatment of Tuberculosis Disease. 7th ed. Public Health Agency of Canada; 2014

35 Nahid P, Dorman SE, Alipanah N, et al. Official American Thoracic Society/Centers for Disease Control and Prevention/Infectious Diseases Society of America Clinical Practice Guidelines: treatment of drug-susceptible tuberculosis. Clin Infect Dis 2016;63 (07):e147-e195

36 Solé A, Part-Lopez M, Saez B, et al. Tuberculosis following lung transplantation. A 27-year Spanish Multicenter Experience. Incidence, presentation, prevention and treatment with rifampicin. Arch Bronconeumol 2020;56(08):493-498

37 Morales P, Briones A, Torres JJ, Solé A, Pérez D, Pastor A. Pulmonary tuberculosis in lung and heart-lung transplantation: fifteen years of experience in a single center in Spain. Transplant Proc 2005;37(09):4050-4055

38 Gomila-Grange A, Pérez-Recio S, Camprubí-Ferrer D, et al. Rifabutin for treating tuberculosis in solid organ transplant recip- ients: a retrospective observational study and literature review. Transpl Infect Dis 2020:e13471

39 Tabarsi P, Farshidpour M, Marjani M, et al. Mycobacterial infection and the impact of rifabutin treatment in organ transplant recipients: a single-center study. Saudi J Kidney Dis Transpl 2015;26(01):6-11

40 Prevots DR, Marras TK. Epidemiology of human pulmonary infection with nontuberculous mycobacteria: a review. Clin Chest Med 2015;36(01):13-34

41 Zweijpfenning SMH, Ingen JV, Hoefsloot W. Geographic distribution of nontuberculous mycobacteria isolated from clinical specimens: a systematic review. Semin Respir Crit Care Med 2018;39(03):336-342

42 Hoefsloot W, van Ingen J, Andrejak C, et al; Nontuberculous Mycobacteria Network European Trials Group. The geographic diversity of nontuberculous mycobacteria isolated from pulmonary samples: an NTM-NET collaborative study. Eur Respir J 2013;42(06):1604-1613

43 Spaulding AB, Lai YL, Zelazny AM, et al. Geographic distribution of nontuberculous mycobacterial species identified among clinical isolates in the United States, 2009-2013. Ann Am Thorac Soc 2017;14(11):1655-1661

44 Al Houqani M, Jamieson F, Chedore P, Mehta M, May K, Marras TK. Isolation prevalence of pulmonary nontuberculous mycobacteria in Ontario in 2007. Can Respir J 2011;18(01):19-24

45 Marras TK, Chedore P, Ying AM, Jamieson F. Isolation prevalence of pulmonary non-tuberculous mycobacteria in Ontario, 1997 2003. Thorax 2007;62(08):661-666

46 Marras TK, Mendelson D, Marchand-Austin A, May K, Jamieson FB. Pulmonary nontuberculous mycobacterial disease, Ontario, Canada, 1998-2010. Emerg Infect Dis 2013;19(11):1889-1891

47 Marras TK, Campitelli MA, Lu H, et al. Pulmonary nontuberculous mycobacteria-associated deaths, Ontario, Canada, 2001-2013. Emerg Infect Dis 2017;23(03):468-476

48 Griffith DE, Aksamit T, Brown-Elliott BA, et al; ATS Mycobacterial Diseases Subcommittee American Thoracic Society Infectious Disease Society of America. An official ATS/IDSA statement: diagnosis, treatment, and prevention of nontuberculous mycobacterial diseases. Am J Respir Crit Care Med 2007;175(04): 367-416

49 Mirsaeidi M, Machado RF, Garcia JGN, Schraufnagel DE. Nontuberculous mycobacterial disease mortality in the United States, 1999-2010: a population-based comparative study. PLoS ONE 2014;9(03):e91879

50 Shah NM, Davidson JA, Anderson LF, et al. Pulmonary Mycobacterium avium-intracellulare is the main driver of the rise in non-tuberculous mycobacteria incidence in England, Wales and Northern Ireland, 2007-2012. BMC Infect Dis 2016;16 (01):195

51 Thomson RMNTM working group at Queensland TB Control Centre and Queensland Mycobacterial Reference Laboratory. Changing epidemiology of pulmonary nontuberculous mycobacteria infections. Emerg Infect Dis 2010;16(10):1576-1583

52 Aubry A, Mougari F, Reibel F, Cambau E. Mycobacterium marinum. Microbiol Spectr 2017;5(02):. Doi: 10.1128/microbiolspec.TNMI7-0038-2016

53 Sander MA, Isaac-Renton JL, Tyrrell GJ. Cutaneous nontuberculous mycobacterial infections in Alberta, Canada: an epidemiologic study and review. J Cutan Med Surg 2018;22(05):479-483

54 Jabbour SF, Malek AE, Kechichian EG, Tomb RR, Nasr MW. Nontuberculous mycobacterial infections after cosmetic procedures: a systematic review and management algorithm. Dermatol Surg 2020;46(01):116-121

55 Leto Barone AA, Grzelak MJ, Frost C, et al. Atypical mycobacterial infections after plastic surgery procedures abroad: a multidisciplinary algorithm for diagnosis and treatment. Ann Plast Surg 2020;84(03):257-262 
56 Rao M, Silveira FP. Non-tuberculous mycobacterial infections in thoracic transplant candidates and recipients. Curr Infect Dis Rep 2018;20(06):14

57 Olivier KN, Weber DJ, Wallace RJ Jr, et al; Nontuberculous Mycobacteria in Cystic Fibrosis Study Group. Nontuberculous mycobacteria. I: Multicenter prevalence study in cystic fibrosis. Am J Respir Crit Care Med 2003;167(06):828-834

58 Luong M-L, Morrissey O, Husain S. Assessment of infection risks prior to lung transplantation. Curr Opin Infect Dis 2010;23(06): 578-583

59 Bernut A, Nguyen-Chi M, Halloum I, Herrmann J-L, Lutfalla G, Kremer L. Mycobacterium abscessus-induced granuloma formation is strictly dependent on TNF signaling and neutrophil trafficking. PLOS Pathog 2016;12(11):e1005986

60 Bernut A, Dupont C, Ogryzko NV, et al. CFTR protects against Mycobacterium abscessus infection by fine-tuning host oxidative defenses. Cell Rep 2019;26(07):1828-1840.e4

61 Furukawa BS, Flume PA. Nontuberculous mycobacteria in cystic fibrosis. Semin Respir Crit Care Med 2018;39(03): 383-391

62 Horsburgh CR Jr, Selik RM. The epidemiology of disseminated nontuberculous mycobacterial infection in the acquired immunodeficiency syndrome (AIDS). Am Rev Respir Dis 1989;139(01): 4-7

63 Henkle E, Winthrop KL. Nontuberculous mycobacteria infections in immunosuppressed hosts. Clin Chest Med 2015;36(01):91-99

64 Browne SK, Burbelo PD, Chetchotisakd P, et al. Adult-onset immunodeficiency in Thailand and Taiwan. $\mathrm{N}$ Engl J Med 2012;367(08):725-734

65 Honda JR, Alper S, Bai X, Chan ED. Acquired and genetic host susceptibility factors and microbial pathogenic factors that predispose to nontuberculous mycobacterial infections. Curr Opin Immunol 2018;54:66-73

66 Sax H, Bloemberg G, Hasse B, et al. Prolonged outbreak of Mycobacterium chimaera infection after open-chest heart surgery. Clin Infect Dis 2015;61(01):67-75

67 Doucette K, Fishman JA. Nontuberculous mycobacterial infection in hematopoietic stem cell and solid organ transplant recipients. Clin Infect Dis 2004;38(10):1428-1439

68 Keating MR, Daly JSAST Infectious Diseases Community of Practice. Nontuberculous mycobacterial infections in solid organ transplantation. Am J Transplant 2013;13(Suppl 4):77-82

69 Todd JL, Lakey J, Howell D, Reidy M, Zaas D. Portal hypertension and granulomatous liver disease in a lung transplant recipient due to disseminated atypical mycobacterial infection. Am J Transplant 2007;7(05):1300-1303

70 Longworth SA, Vinnard C, Lee I, Sims KD, Barton TD, Blumberg EA. Risk factors for nontuberculous mycobacterial infections in solid organ transplant recipients: a case-control study. Transpl Infect Dis 2014;16(01):76-83

71 Johnson MM, Odell JA. Nontuberculous mycobacterial pulmonary infections. J Thorac Dis 2014;6(03):210-220

72 Prince DS, Peterson DD, Steiner RM, et al. Infection with Mycobacterium avium complex in patients without predisposing conditions. N Engl J Med 1989;321(13):863-868

73 Sexton P, Harrison AC. Susceptibility to nontuberculous mycobacterial lung disease. Eur Respir J 2008;31(06):1322-1333

74 Dorman S, Subramanian AAST Infectious Diseases Community of Practice. Nontuberculous mycobacteria in solid organ transplant recipients. Am J Transplant 2009;9(Suppl 4):S63-S69

75 Patel R, Roberts GD, Keating MR, Paya CV. Infections due to nontuberculous mycobacteria in kidney, heart, and liver transplant recipients. Clin Infect Dis 1994;19(02):263-273

76 Abad CL, Razonable RR. Non-tuberculous mycobacterial infections in solid organ transplant recipients: an update.J Clin Tuberc Other Mycobact Dis 2016;4:1-8

77 Haworth CS, Banks J, Capstick T, et al. British Thoracic Society guidelines for the management of non-tuberculous mycobacte- rial pulmonary disease (NTM-PD). Thorax 2017;72(Suppl 2): ii1-ii64

78 Longworth SA, Daly JSAST Infectious Diseases Community of Practice. Management of infections due to nontuberculous mycobacteria in solid organ transplant recipients-guidelines from the American Society of Transplantation Infectious Diseases Community of Practice. Clin Transplant 2019;33(09): e13588

79 Paterson DL, Singh N. Interactions between tacrolimus and antimicrobial agents. Clin Infect Dis 1997;25(06):1430-1440

80 De La Cruz O, Minces LR, Silveira FP. Experience with linezolid for the treatment of nocardiosis in organ transplant recipients. J Infect 2015;70(01):44-51

81 Tessier JM, Puzio T, Young A, Wolfe L, Han J, Duane TM. Thrombocytopenia associated with linezolid therapy in solid organ transplant recipients: a retrospective cohort study. Surg Infect (Larchmt) 2015;16(04):361-367

82 Bidell MR, Lodise TP. Fluoroquinolone-associated tendinopathy: Does levofloxacin pose the greatest risk? Pharmacotherapy 2016;36(06):679-693

83 Anjan S, Morris MI. How can we improve the outcome for transplant patients with nontuberculous mycobacterial infections? Future Microbiol 2018;13(08):903-914

84 Aslam S. Bacteriophage therapy as a treatment option for transplant infections. Curr Opin Infect Dis 2020;33(04):298-303

85 Aslam S, Courtwright AM, Koval C, et al. Early clinical experience of bacteriophage therapy in 3 lung transplant recipients. Am J Transplant 2019;19(09):2631-2639

86 Dedrick RM, Guerrero-Bustamante CA, Garlena RA, et al. Engineered bacteriophages for treatment of a patient with a disseminated drug-resistant Mycobacterium abscessus. Nat Med 2019; 25(05):730-733

87 Fox A. Engineered phages stymie drug-resistant infection. Science 2019;364(6440):518-519

88 Weill D. Lung transplantation: indications and contraindications. J Thorac Dis 2018;10(07):4574-4587

89 Friedman DZP, Cervera C, Halloran K, Tyrrell G, Doucette K. Nontuberculous mycobacteria in lung transplant recipients: prevalence, risk factors, and impact on survival and chronic lung allograft dysfunction. Transpl Infect Dis 2020;22(02):e13229

90 Hamad Y, Pilewski JM, Morrell M, D'Cunha J, Kwak EJ. Outcomes in lung transplant recipients with Mycobacterium abscessus infection: a 15-year experience from a large tertiary care center. Transplant Proc 2019;51(06):2035-2042

91 Longworth SA, Blumberg EA, Barton TD, Vinnard C. Non-tuberculous mycobacterial infections after solid organ transplantation: a survival analysis. Clin Microbiol Infect 2015;21(01): 43-47

92 Huang HC, Weigt SS, Derhovanessian A, et al. Non-tuberculous mycobacterium infection after lung transplantation is associated with increased mortality. J Heart Lung Transplant 2011;30(07): 790-798

93 Knoll BM, Kappagoda S, Gill RR, et al. Non-tuberculous mycobacterial infection among lung transplant recipients: a 15-year cohort study. Transpl Infect Dis 2012;14(05):452-460

94 George IA, Santos CAQ, Olsen MA, Bailey TC. Epidemiology and outcomes of nontuberculous mycobacterial infections in solid organ transplant recipients at a Midwestern center. Transplantation 2016;100(05):1073-1078

95 Shah SK, McAnally KJ, Seoane L, et al. Analysis of pulmonary nontuberculous mycobacterial infections after lung transplantation. Transpl Infect Dis 2016;18(04):585-591

96 Hirama T, Singer LG, Brode SK, Marras TK, Husain S. Outcomes of a peri- and postoperative management protocol for non-TB mycobacteria in lung transplant recipients. Chest 2020;158 (02):523-528

97 Kabbani D, Kozlowski HN, Cervera C, et al. Granuloma in the explanted lungs: Infectious causes and impact on post-lung 
transplant mycobacterial infection. Transpl Infect Dis 2020;22 (03):e13262

98 Watkins RR, Lemonovich TL. Evaluation of infections in the lung transplant patient. Curr Opin Infect Dis 2012;25(02):193-198

99 Taylor JL, Palmer SM. Mycobacterium abscessus chest wall and pulmonary infection in a cystic fibrosis lung transplant recipient. J Heart Lung Transplant 2006;25(08):985-988

100 Chernenko SM, Humar A, Hutcheon M, et al. Mycobacterium abscessus infections in lung transplant recipients: the international experience. J Heart Lung Transplant 2006;25(12): 1447-1455

101 Zaidi S, Elidemir O, Heinle JS, et al. Mycobacterium abscessus in cystic fibrosis lung transplant recipients: report of 2 cases and risk for recurrence. Transpl Infect Dis 2009;11(03):243-248

102 Perez AA, Singer JP, Schwartz BS, et al. Management and clinical outcomes after lung transplantation in patients with pre-transplant Mycobacterium abscessus infection: a single center experience. Transpl Infect Dis 2019;21(03):e13084

103 Brown-Elliott BA, Wallace RJ Jr. Clinical and taxonomic status of pathogenic nonpigmented or late-pigmenting rapidly growing mycobacteria. Clin Microbiol Rev 2002;15(04):716-746

104 Trulock EP, Bolman RM, Genton R. Pulmonary disease caused by Mycobacterium chelonae in a heart-lung transplant recipient with obliterative bronchiolitis. Am Rev Respir Dis 1989;140 (03):802-805

105 Swetter SM, Kindel SE, Smoller BR. Cutaneous nodules of Mycobacterium chelonae in an immunosuppressed patient with preexisting pulmonary colonization. J Am Acad Dermatol 1993;28 (2, Pt 2):352-355

106 Baisi A, Nosotti M, Chella B, Santambrogio L. Relapsing cutaneous Mycobacterium chelonae infection in a lung transplant patient. Transpl Int 2005;18(09):1117-1119

107 Ambaraghassi G, Ferraro P, Poirier C, Rouleau D, Fortin C. Double lung transplantation in an HIV-positive patient with Mycobacterium kansasii infection. Transpl Infect Dis 2019;21(01):e12999

108 Malouf MA, Glanville AR. The spectrum of mycobacterial infection after lung transplantation. Am J Respir Crit Care Med 1999; 160(5, Pt 1):1611-1616

109 Ariza-Heredia EJ, Dababneh AS, Wilhelm MP, Wengenack NL, Razonable RR, Wilson JW. Mycobacterium wolinskyi: a case series and review of the literature. Diagn Microbiol Infect Dis 2011;71(04):421-427

110 Date A, John GT, Thomas PP, Jacob CK. Leprosy and renal transplantation. Lepr Rev 1998;69(01):40-45

111 Guditi S, Ram R, Ismal KM, et al. Leprosy in a renal transplant recipient: review of the literature. Transpl Infect Dis 2009;11 (06):557-562

112 Modi K, Mancini M, Joyce MP. Lepromatous leprosy in a heart transplant recipient. Am J Transplant 2003;3(12):1600-1603
113 Gasink LB, Seymour C, Blumberg EA, Goldberg LR, Fishman NO. An uncommon presentation of an uncommon disease: leprosy in a heart transplant recipient. J Heart Lung Transplant 2006;25 (07):854-856

114 World Health Organization. Guidelines for the Diagnosis, Treatment and Prevention of Leprosy. Published online 2018 Accessed March 5, 2021 at: https://www.who.int/lep/resources/9789290226383/en/

115 Finch CK, Chrisman CR, Baciewicz AM, Self TH. Rifampin and rifabutin: an update. Arch Intern Med 2002;162:985-992

116 Sparkes T, Lemonovich TLAST Infectious Diseases Community of Practice. Interactions between anti-infective agents and immunosuppressants-Guidelines from the American Society of Transplantation Infectious Diseases Community of Practice. Clin Transplant 2019;33(09):e13510

117 Hernández-Garduño E, Rodrigues M, Elwood RK. The incidence of pulmonary non-tuberculous mycobacteria in British Columbia, Canada. Int J Tuberc Lung Dis 2009;13(09): 1086-1093

118 Bodle EE, Cunningham JA, Della-Latta P, Schluger NW, Saiman L. Epidemiology of nontuberculous mycobacteria in patients without HIV infection, New York City. Emerg Infect Dis 2008;14(03): 390-396

119 Prevots DR, Shaw PA, Strickland D, et al. Nontuberculous mycobacterial lung disease prevalence at four integrated health care delivery systems. Am J Respir Crit Care Med 2010;182(07): 970-976

120 Smith GS, Ghio AJ, Stout JE, et al. Epidemiology of nontuberculous mycobacteria isolations among central North Carolina residents, 2006-2010. J Infect 2016;72(06):678-686

121 Henkle E, Hedberg K, Schafer S, Novosad S, Winthrop KL. Population-based Incidence of pulmonary nontuberculous mycobacterial disease in Oregon 2007 to 2012. Ann Am Thorac Soc 2015;12(05):642-647

122 Winthrop KL, McNelley E, Kendall B, et al. Pulmonary nontuberculous mycobacterial disease prevalence and clinical features: an emerging public health disease. Am J Respir Crit Care Med 2010;182(07):977-982

123 Cassidy PM, Hedberg K, Saulson A, McNelly E, Winthrop KL. Nontuberculous mycobacterial disease prevalence and risk factors: a changing epidemiology. Clin Infect Dis 2009;49(12): e124-e129

124 Novosad SA, Henkle E, Schafer S, et al. Mortality after respiratory isolation of nontuberculous mycobacteria: a comparison of patients who did and did not meet disease criteria. Ann Am Thorac Soc 2017;14(07):1112-1119

125 Adjemian J, Frankland TB, Daida YG, et al. Epidemiology of nontuberculous mycobacterial lung disease and tuberculosis, Hawaii, USA. Emerg Infect Dis 2017;23(03):439-447 